\title{
Causes of scatter in high rate fracture testing of polymers
}

\author{
Authors: S. Agnelli ${ }^{1}$, I. Horsfall ${ }^{2}$ \\ ${ }^{1}$ Università degli Studi di Brescia, Dipartimento di Ingegneria Meccanica e Industriale, via Branze 38, 25123 Brescia, Italy \\ ${ }^{2}$ Cranfield University, Department of Engineering and Applied Science, Shrivenham, Swindon, SN6 8LA, United Kingdom
}

Corresponding author: Tel.: +39030 3715925, Fax: +39 030 3702448. E-mail address: silvia.agnelli@ing.unibs.it (S. Agnelli)

\begin{abstract}
A method based on the measurement of failure time is being developed by the European Structural Integrity Society to determine high rate fracture toughness of polymers. The test method appears reasonably straightforward but produced unacceptably high scatter, due to failure time data scatter. In this work an experimentally based sensitivity study was performed by fracture tests at $1 \mathrm{~ms}^{-1}$ in order to determine the causes of scatter and to seek to improve the test protocol. No single cause of scatter was identified but the quality and repeatability of the notching technique was identified as a major contributor.
\end{abstract}

\section{Keywords}

Impact fracture; polymers; dynamic fracture; toughness testing

\section{Introduction}

A specific protocol for determining fracture toughness of polymers at high loading rates $\left(>1 \mathrm{~ms}^{-1}\right)$ does not yet exist, but is needed for two reasons: firstly, polymers are viscoelastic materials, therefore their properties are rate-dependent and must be measured at speeds characteristic of the in service loading; secondly because the dynamic effects causing oscillations in the load signal are not accounted for in existing lower rate test [1]. Dynamic effects can be controlled at moderately high loading rates by damping techniques, as suggested in the ISO 17281 standard, which allows measurement of fracture toughness of polymers at loading rates lower than $1 \mathrm{~ms}^{-1}$ [1]. At rates higher than $1 \mathrm{~ms}^{-1}$ approaches based on the loading record are unsuitable because most of the load is absorbed by the momentum, and the time to fracture is therefore comparable with the time taken by the stress waves to travel across the specimen, causing oscillations that make it impossible to define the point of fracture initiation.

To avoid the necessity of load measurement to evaluate fracture toughness, a methodology is being developed by Technical Committee 4 (TC4) of the European Structural Integrity Society (ESIS) 
which requires only the measurement of time to fracture. This approach is based on the dynamic key curves concept proposed by Böhme [2]. The basic assumption of the dynamic key curves method is that dynamic stress intensity factor, $\mathrm{K}_{\mathrm{Id}}(\mathrm{t})$, which is related to the real, dynamic crack tip loading, can be separated into a quasi-static part, $\mathrm{K}_{\mathrm{st}}(\mathrm{t})$, and a dynamic correction function, $\mathrm{k}_{\mathrm{d}}(\mathrm{t})$, which describes the influence of the transient dynamic effects, as shown by the following relationship:

$\mathrm{K}_{\mathrm{Id}}(\mathrm{t})=\mathrm{K}_{\mathrm{st}}(\mathrm{t}) \cdot \mathrm{k}_{\mathrm{d}}(\mathrm{t})$

The quasi-static part can be analytically derived from a simple mass-spring model and calculated by the following equation:

$$
\mathrm{K}_{\mathrm{st}}=\frac{\mathrm{f}}{\varphi} \cdot \frac{\mathrm{E}}{\mathrm{W}^{\frac{1}{2}}} \cdot \frac{\mathrm{Vt}}{\left(1+\frac{1}{\alpha}\right)}
$$

where $\mathrm{E}$ is the Young's modulus, $\mathrm{V}$ is the impact velocity, $\mathrm{t}$ is the duration of the specimen loading, $\alpha$ is the ratio of contact stiffness to specimen stiffness, $f$ is the geometry factor according to Srawley [3], $\mathrm{W}$ is the specimen width and $\varphi$ is Bucci's formula for dimensionless specimen compliance [4]. The dynamic correction factor can be pre-determined in different ways; experimental [2], analytical [5] or numerical [6]. This term is represented by different functions, dependent on the loading device, specimen geometry and elastic modulus of the material, which in a normalized form are called dynamic key curves (DKC). If the proper dynamic key curve is known for the configuration used, $\mathrm{K}_{\mathrm{Id}}$ value at fracture initiation $\left(\mathrm{K}_{\mathrm{Ic}}\right)$ can be estimated by evaluating both terms, $\mathrm{K}_{\mathrm{st}}$ and $\mathrm{k}_{\mathrm{d}}$, from the time to fracture, $\mathrm{t}_{\mathrm{f}}$, measured in a fracture test.

Two round robin testing activities have been carried out by ESIS TC4 to develop this approach. In the second of these test series, completed in 2008, a testing procedure was adopted in which the failure time was measured using enly the signal of a strain gauge placed near the crack tip. This activity was carried out on three different polymeric materials (PVC, PMMA and HDPE), by five laboratories, at various speeds ranging from 0.2 to $27 \mathrm{~ms}^{-1}$. The DKC based approach turned out to be a promising methodology, but its accuracy was weakened by a high degree of scatter in $\mathrm{K}_{\mathrm{Id}}$ results, even in the relatively slow tests. This scatter existed both within data sets from individual laboratories and between the data sets of different laboratories so it could not be explained entirely by differences in equipment or technique. By analysing data of each single variable of Equations 1 and 2, time to failure results were identified as the most likely cause of scatter. Poor repeatability of results and the fact that data scatter was not velocity dependent suggested that a flaw in the experimental approach used for time measurement was more likely. 
The aim of the present work is to determine the reason for the large degree of scatter affecting failure time results and, if possible, to provide recommendations and improvements which might be included in a further round robin activity. Several factors, possible sources of scatter, are investigated and an attempt is made to establish which factors influence the failure time measurements. In the present work an analysis of the procedure proposed in the round robin test for failure time measurement is shown, which is performed both by analysing results and measurements collected during the round robin activity and by performing tests on new specimens.

\section{Experimental}

The same testing procedure used for the second round robin activity and indicated in the protocol draft (see Appendix A in [6]) was adopted to perform tests on new specimens. The aim behind this was to recreate the same experimental conditions that led to the scattered results of the original round robin. The experimental details of the procedure, adopted in this work are briefly described below. Any modification made in this work with reference to the draft protocol is highlighted.

The same polymeric materials used in the second round robin activity, PVC and PMMA, were tested. For that test specimens were centrally machined and notched, and then delivered to several laboratories for testing. In the current work the specimens were machined by the same institute and notched by similar notching techniques (but different operator). The tests were performed on machined Single Edge Notched in Bending (SEB) specimens of thickness $8 \mathrm{~mm}$, width (W) $16 \mathrm{~mm}$, length to width ratio-5.5, span to width ratio 4 and initial crack length to width ratio (a/W) 0.3 . Dimensional tolerances of the machined specimen (width and thickness) were $0.1 \mathrm{~mm}$ for PVC and $0.05 \mathrm{~mm}$ for PMMA specimens. Notches were first machined, then sharpened (pre-cracked) by different techniques, due to the differences in pre-cracking behaviour between the materials. Notches in PVC samples were sharpened by sliding an industrial razor blade with a tip radius of 13 $\mu \mathrm{m}$ at the root of the notch (sliding technique), while notches in PMMA samples were sharpened by the wedging action of a blade tapped into the notch (tapping technique). Fig. 1 shows two examples of a typical pre-crack in a PMMA (Fig. 1a, c) and in a PVC sample (Fig. 1b, d). Fig 1a and 1b are lateral views, while Fig. 1c and 1d show the fracture surfaces of tested samples. PVC pre-cracks were quite short (some tenth of millimetre) but the crack front was straight, while PMMA tapped notches were longer, sharper and characterised by curved crack fronts that in some cases were also asymmetrical and not in a flat plane. The notch tip radius, although not quantified, was assumed to be constant along the crack front. The initial crack length was measured directly on the fracture surfaces of the specimens after the test. As indicated by the protocol, the crack length was the average over five lengths measured at five equidistant positions over the thickness of the specimen. 
The initial crack length to width ratio (a/W) of the PVC specimens was in the range of $0.3 \pm 0.02$, while that of PMMA was more dispersed, ranging from 0.30 to 0.35 . In addition the crack length in PMMA specimens varied across the specimen. The difference between the minimum and maximum crack length measured on the same specimen averaged $0.61 \mathrm{~mm}$ (corresponding to an error of a/W of 0.03 ), while the highest difference measured was 0.93 (corresponding to a variation of a/W of 0.06). For PVC specimens the average difference between the minimum and maximum crack length measured on the same specimen was much lower, $0.12 \mathrm{~mm}$, corresponding to an a/W ratio variability lower than 0.01 .

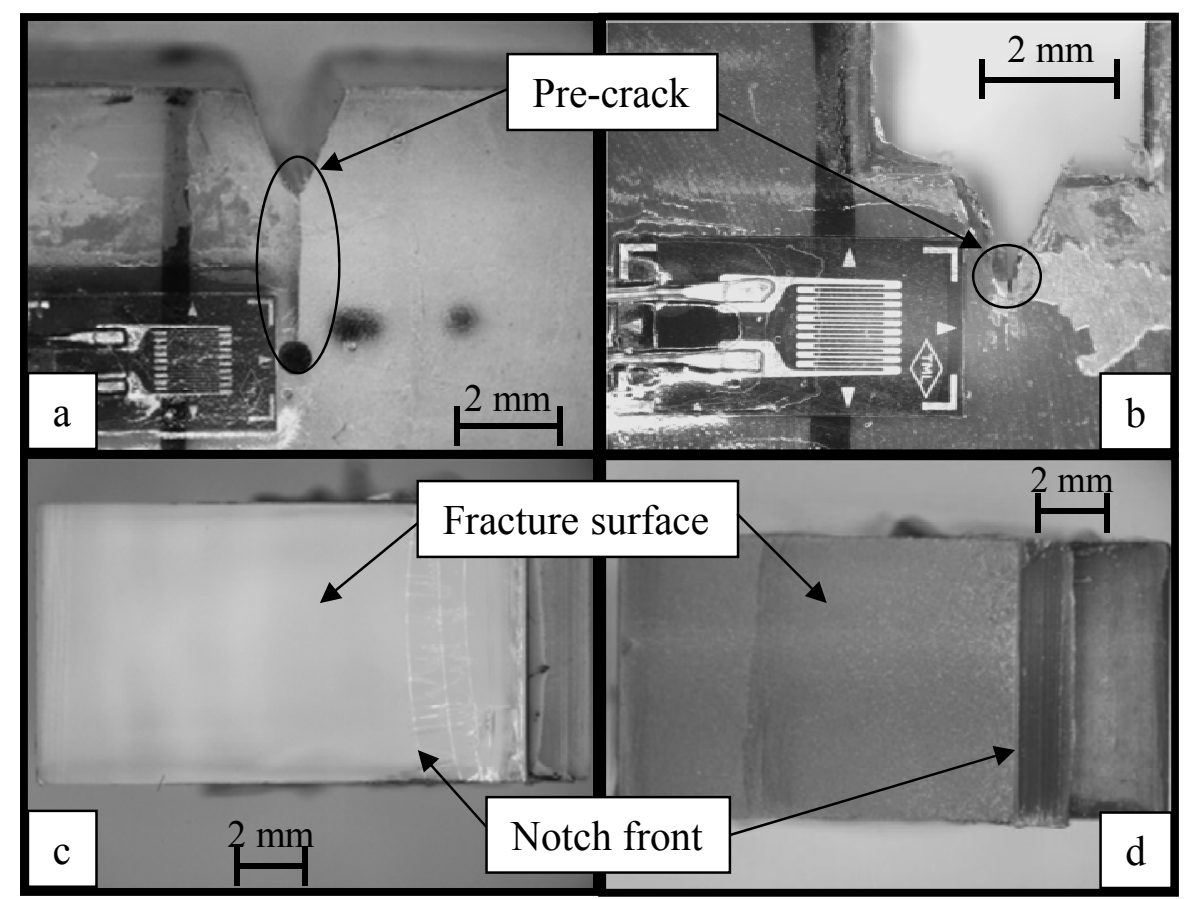

Fig. 1: a) c) PMMA and b) d) PVC specimens notches and pre-cracks: a) b) lateral views, c) d) fractured surfaces

In the current work some PVC and PMMA specimens were notched by a third technique: a commercial cutting machine (CEAST Notchvis) was used and the blade geometry was arranged such that material was cut and removed from the notch in small amounts over a large number of cutting strokes (further details will be provided in the results section). This differed from the round robin in which pre cracking in PVC specimens was achieved by a blade which was pushed through the notch only one or two times and did not necessarily remove material. At least one strain gauge, with a grid size smaller than $1.5 \times 1.5 \mathrm{~mm}^{2}$, was bonded as close to the notch tip of each test piece as possible (as shown in Fig. 1a), as recommended by the draft protocol, and the signal was amplified by a high bandwidth strain gauge amplifier to meet the protocol requirements. 
Tests were performed by a horizontal impact machine, a prototype developed by Imatek $^{1}$ in which the carriage carrying the striker is accelerated by a linear induction motor which then disconnects from the carriage prior to impact. This testing machine fulfilled the requirements stated by the draft protocol (i.e.: minimum loading rate capacity, minimum impact energy and speed measurement accuracy). Aside from the protocol procedure, in this work tests were also recorded by a high speed video camera running at typically 150,000 frames per second, this was used to provide an independent measure of time to failure to check the reliability of the strain gauge signal. In order to have the largest possible number of useful frames, tests were carried out at impact speeds at the lower end of those performed in the round robin, $1 \mathrm{~ms}^{-1}$, and only few samples were tested at $4 \mathrm{~ms}^{-1}$. The protocol draft recommends to keep the test piece in place with rubber bands, but since the only reason for this was to prevent it moving before the impact due to vibrations, the rubber bands were replaced in this work by adhesive tape strips.

\section{Results and discussion}

Time to failure was experimentally measured, according to the protocol, by the signal of an uncalibrated strain gauge bonded to the specimen. Fig. 2 represents an example of a typical strain gauge trace versus time from a PVC sample tested at $1 \mathrm{~ms}^{-1}$. Two vertical lines are traced in Fig. 2: one where the strain gauge signal starts to rise, corresponding to the start of the crack tip loading, and one at the highest peak of the curve, corresponding to fracture initiation. According to the round robin procedure, time to fracture is evaluated as the interval between these two points.

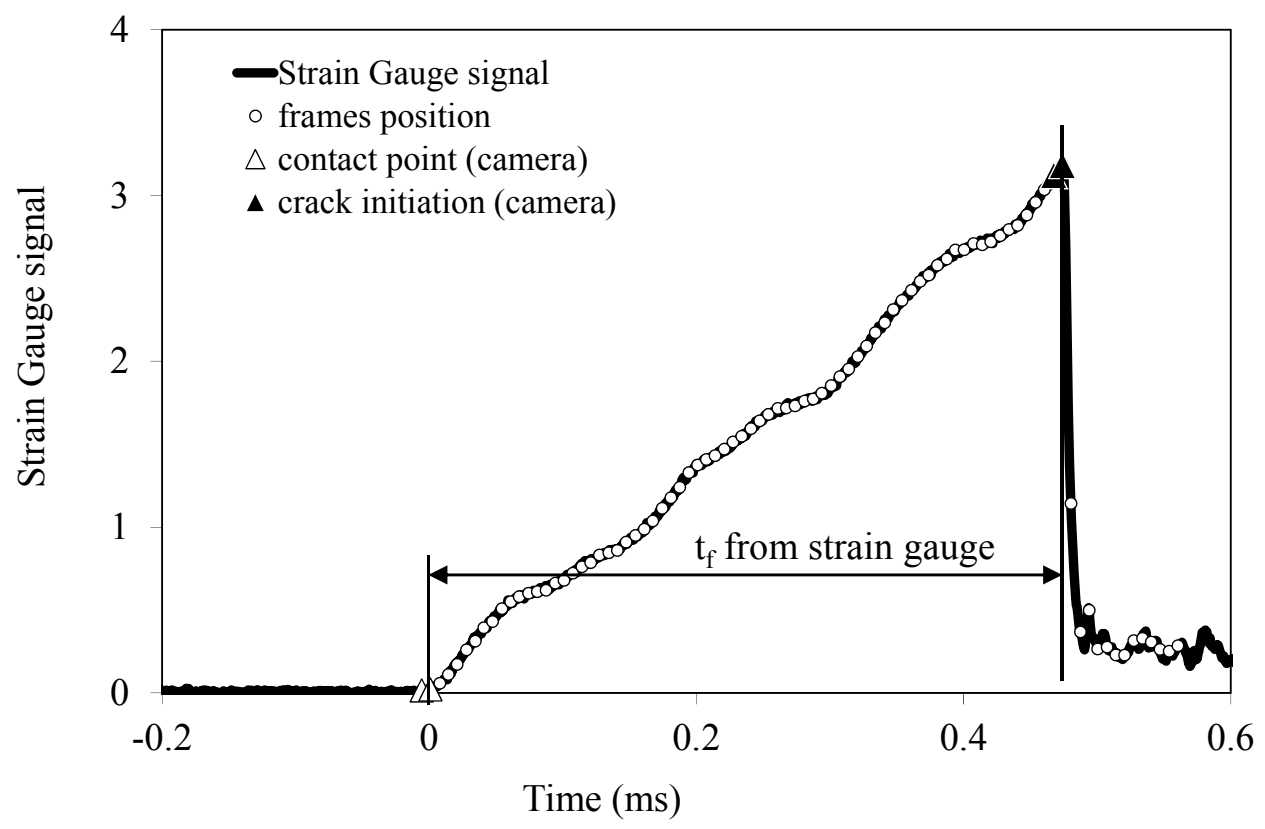

Fig. 2: Strain gauge signal of an impact test at $1 \mathrm{~ms}^{-1}$ on a PVC SE(B) specimen

\footnotetext{
${ }^{1}$ Imatek Ltd, Hertfordshire UK.
} 
The open circles in Fig. 2 on the strain gauge curve indicate times at which the frames were recorded by the high speed camera. The first point of contact between striker and specimen and the fracture initiation events were observed on the video and the times of the corresponding frames are indicated with triangles on the strain curve. It is apparent that the time to failure observed from the high speed video and that from the strain gauge curves are equivalent. The reliability of the strain curve for the measurement of $t_{f}$ was confirmed for all the tests performed, on both PVC and PMMA specimens.

The investigation performed in this work is based on the following observations: $t_{f}$ data scatter affected both materials, was velocity independent and was present within sets of samples from individual laboratories. Since the amount of data was not enough to perform a statistical analysis, scatter of results was highlighted by plotting the normalized failure time, defined as follows:

Normalized failure time $=\frac{t_{f}}{t_{f}(\text { average })} \cdot 100$

where $t_{f}$ (average) is the average over $t_{f}$ of the considered data set. Within each set, the larger the difference between the maximum and the minimum normalized failure time, the higher is the scatter of the data.

Normalized failure times obtained at $1 \mathrm{~ms}^{-1}$ are compared in Fig. 3 for PVC samples and in Fig 4 for PMMA samples. On the abscissa of Fig. 3 and 4 the test sets are displayed. From left to right the first five sets are from the laboratories participating in the second ESIS TC4 round robin activity. It is worth noting that scatter for PMMA is double the scatter of the PVC results.

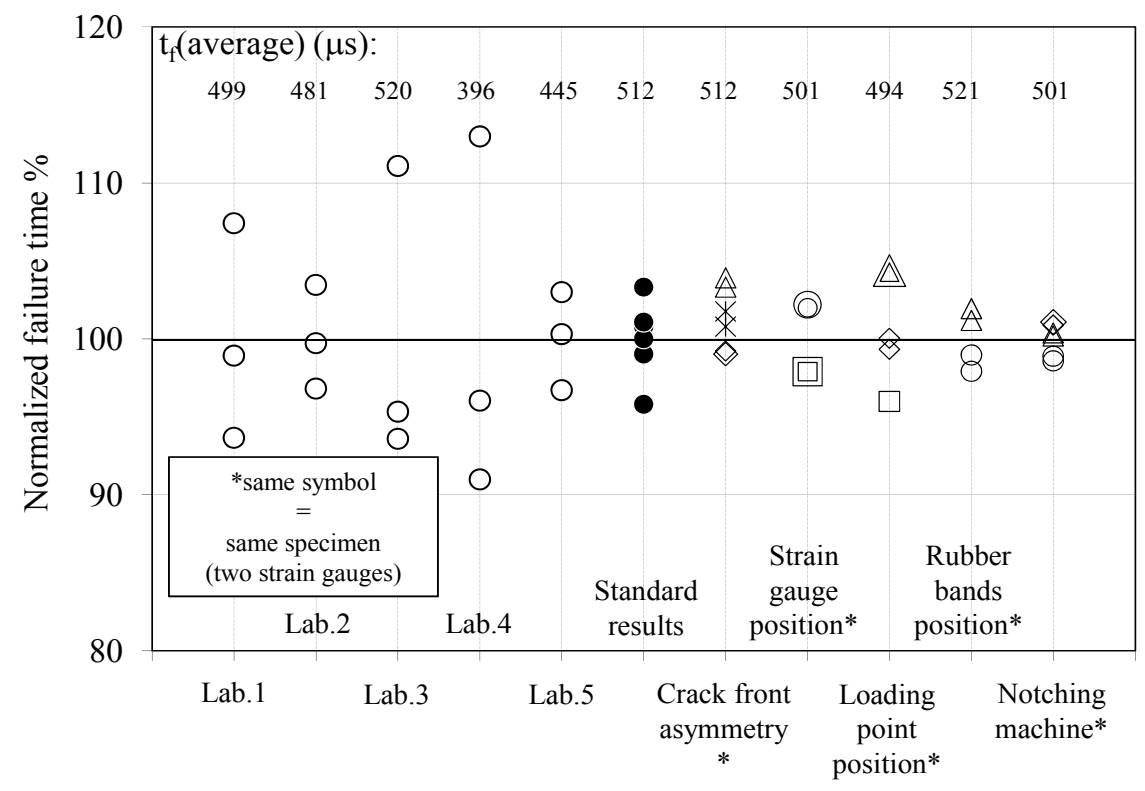

Fig. 3: Normalized time to failure of PVC samples tested at $1 \mathrm{~ms}^{-1}$ 
In order to find the reasons for the high variability of the round robin results, data, measurements and available specimens collected at the end of the round robin activity were analysed. However analysis of the raw data from the round robin showed that there did not appear to be any correlation of the scatter with variations in the initial crack length or the test speed, therefore these two parameters were rejected as possible sources of scatter. Another reason which might have affected failure time repeatability is the penetration of strain gauges adhesive into the pre-crack, but microscopy of fracture surfaces of specimens from laboratory 1 showed no trace of adhesive on the surfaces. at $1 \mathrm{~ms}^{-1}$

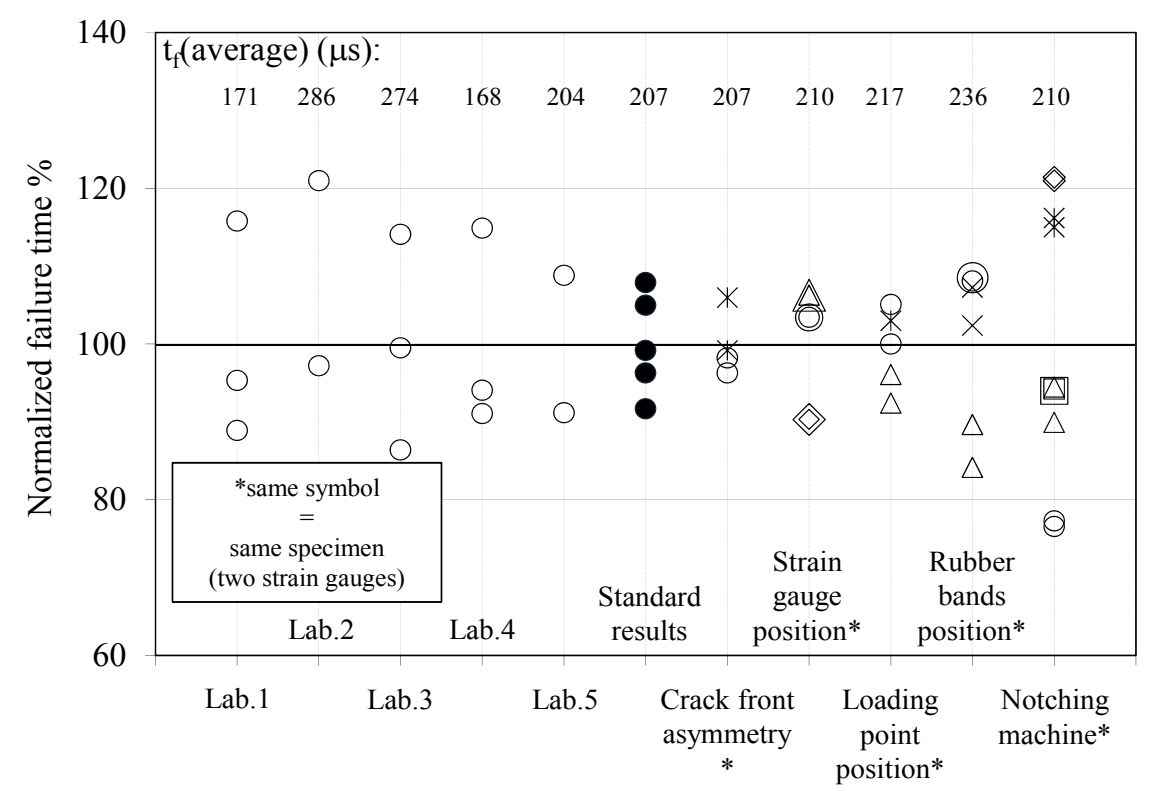

Fig. 4: Normalized time to failure of PMMA samples tested at $1 \mathrm{~ms}^{-1}$

Since not much information could be obtained from the original results of the round robin, some additional experimental tests were performed, at first according to the experimental procedure of the round robin tests [6], described in the Experimental section, to simulate those tests. Standard results are the data obtained in this work by strictly applying the draft protocol. Compared to the results obtained by the round robin activity, Standard results of both materials show a degree of scatter similar to the lowest degree produced by some laboratories of the round robin. In order to identify the causes of the variability of the round robin results, several sets of tests were performed either by introducing modifications to the experimental procedure, or by intentionally introducing an experimental error. Not enough tests were performed to analyse the trend of the results variability as a function of the error magnitude. However, the magnitude of introduced errors was not unreasonably exaggerated, but was such to simulate a reasonable mistake which could be produced by a laboratory participating in a round robin activity. When scatter is significantly modified by 
such errors, then it can be deduced that the factor might have played a role in causing the scatter in the round robin results. The following possible causes for data scatter are investigated:

\subsection{Crack front asymmetry}

Sometimes the crack front was not even through the thickness of a specimen, particularly in PMMA test pieces due to the tapping technique. Therefore the two lateral surfaces of some samples had different actual initial crack length (see Fig. 5a, showing an asymmetric crack front in a PMMA specimen) and this might cause the fracture to initiate at different times depending on the surface considered. To examine this effect two strain gauges were bonded to the opposite lateral surfaces of the test pieces. The results from two strain gauges on the same test piece (within the same set) are displayed in Fig. 3 and 4 with the same symbol shape. It can be seen that in PVC samples there is no difference between the results obtained from the two surfaces, while in PMMA the crack front asymmetry does have an influence, as can be seen from the distance between the coupled points. The crack front asymmetry effects can be observed not only in this set, but also in Loading point position and Rubber bands position sets, by the comparison of points with the same symbol.

\subsection{Strain gauge position}

The distance between the crack tip and the strain gauge might influence time to fracture measurement. In this set two strain gauges were bonded to the same surface of each sample, but at different distances from the crack tip, either perpendicularly or parallel to the loading direction (see Fig. $5 \mathrm{c}$ and $5 \mathrm{~d}$, respectively). The offset distance between two strain gauges on the same specimen ranged between 0.5 and $1 \mathrm{~mm}$. It was found that this distance modifies the magnitude of the strain signal, but not the failure time: in fact in Fig. 3 and 4 symbols of the Strain gauge position set with the same shape (i.e. from strain gauges on the surface of the same sample) are almost superimposed.

\subsection{Loading point position}

A further suspected reason for data scatter is the striker-specimen contact position on the test piece. The contact between striker and specimen can take place out of the cracked, middle section of the sample, depending on the operator skills in positioning the specimen and on lateral shifts of the striker and carriage. Tests for this set were performed by misaligning the sample out of the striker path. Fig. 5e and 5f show, as an example, a frame of the videos taken during tests on PVC specimens, showing the striker approaching the specimen in correspondence (Fig. 5e) or not (Fig. 5f) of the pre-crack. The position of loading on the specimen, although difficult to precisely quantify, was shifted from the middle section no more than $1 \mathrm{~mm}$. The normalized failure times of 
this set show approximately the same scatter as the Standard results set, but this parameter might have a stronger influence if misalignment was increased. An insufficient number of tests was performed to analyse the trend of data scatter as a function of the distance of the position of loading from the middle section. As it was not possible to evaluate the striker-specimen relative position of the testing machines used in the round robin tests, it was difficult to assess whether this parameter increased the degree of scatter in these tests.

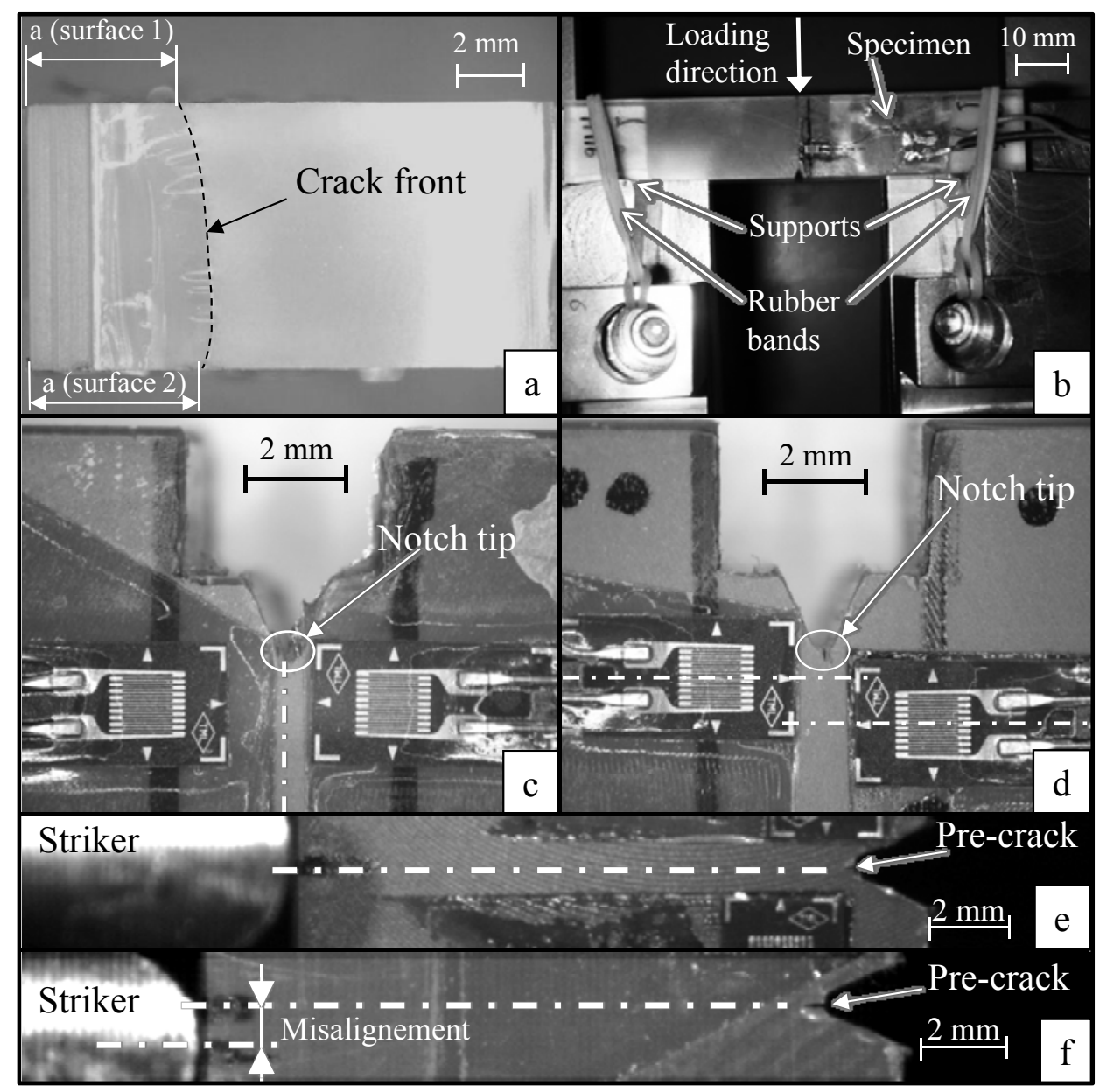

Fig 5: Pictures showing the investigation of some causes of scatter: (a) fracture surface of a PMMA specimen showing an asymmetric crack front; (b)testing configuration with rubber bands placed outside the support span; ( $c$ and d) double strain gauges bonded to the same specimen at different distances form the crack tip, either perpendicularly (c) or parallel (d) to the loading direction; (e and f) frame of the video taken during a test on PVC specimen, showing the striker approaching the specimen in correspondence (e) or not (f) of the pre-crack. 


\subsection{Rubber bands position}

The protocol draft recommends that the specimen is kept in place on the supports by rubber bands. However, if the rubber bands do not press the specimen directly opposite the supports on the surface of the specimen, the crack tip will be pre-loaded. To verify if this condition could alter the time to failure in this set, tests were performed on specimens kept in place by rubber bands at positions inside or outside the support span. Fig. $5 \mathrm{~b}$ shows an example of testing configuration with rubber bands placed outside the support span. The results show that rubber bands position can slightly influence the time to failure in both materials. This effect can be observed in Fig. 4 by comparing the scatter of Rubber bands position and Standard results PMMA sets. The influence of this parameter is not strong enough to produce a scatter comparable to that obtained in the round robin tests.

The high data scatter of round robin results could not be reproduced by analysing any single one of the factors above indicated. However since some of the causes investigated have a slight effect on data scatter (see Crack front asymmetry, Loading point position and Rubber bands position), it is suspected that several factors together contributed to increase data scatter. The pre-cracking technique could be another factor influencing scatter in time to failure. In fact, results of Crack front asymmetry set for PMMA specimens indicate that the variability in the crack front produced by the tapping technique influences the $t_{\mathrm{f}}$ data. For PVC specimens, since the notch was sharpened by sliding an industrial razor blade at the root of the notch without removing material, local compression or deformation could have been introduced into the pre-crack. Therefore, one last set of specimens was prepared using a different notching technique.

\subsection{Notching machine}

Both PMMA and PVC test pieces were notched via a scalpel sliding operation by a CEAST notching machine. The notch (pre-crack) was produced through many passes of an industrial razor blade (tip radius: $13 \mu \mathrm{m}$ ), which cuts a very thin slice of material each step until the desired crack length is reached. Approximately 5 slices per second are taken and the specimens were cooled by compressed air during this process. Fig. 6 shows the notches produced from a lateral view $(a, b)$ and from the fractured surface $(c, d)$ : this technique yields straight crack fronts but in PMMA specimens yields a larger crack tip radius (see Fig. 6b) than that produced by the tapping technique (see Fig. 1a). 


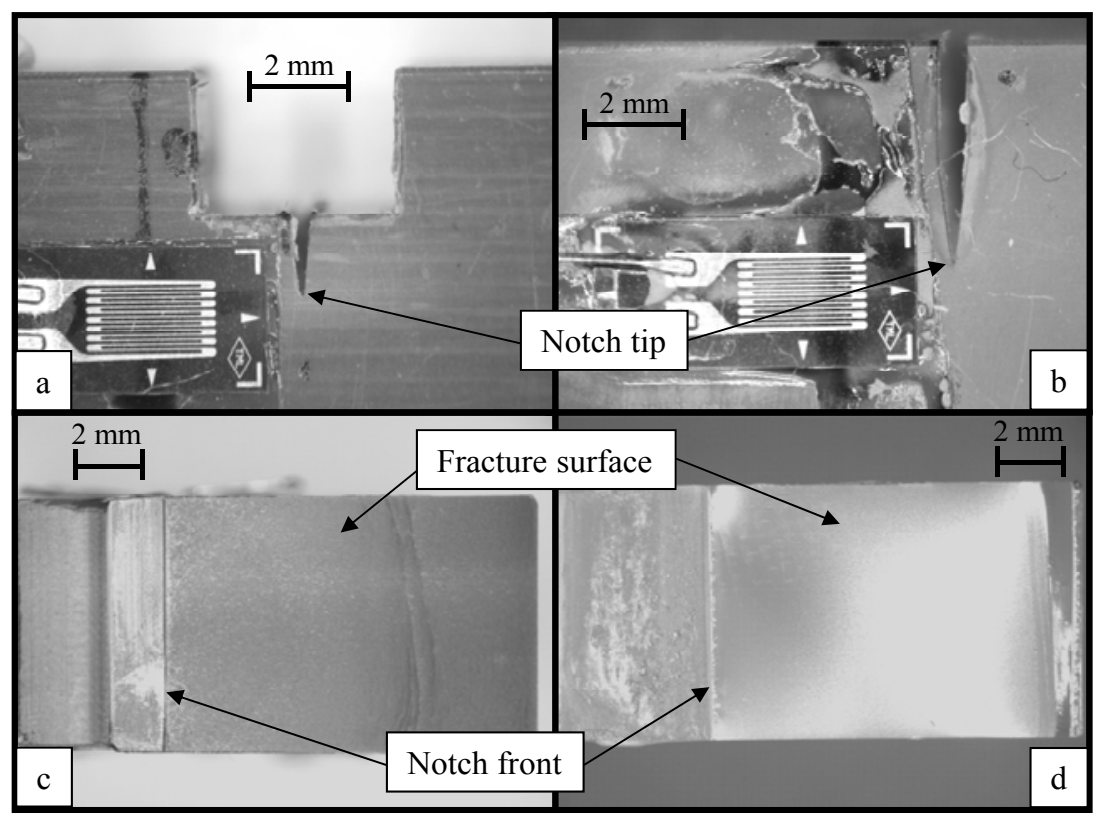

Fig. 6: (a and c) PVC and (b and d) PMMA notched by notching machine specimens: (a and b) lateral views, (c and d) fractured surfaces

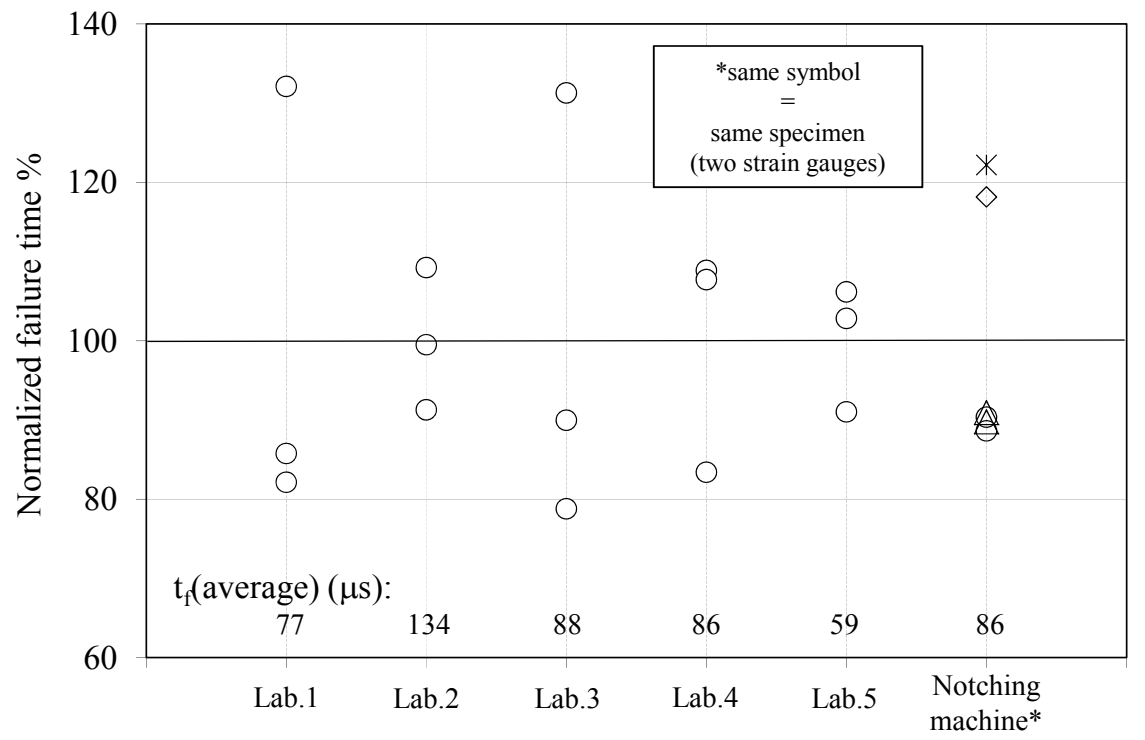

Fig. 7: Normalized time to failure of PVC samples tested at $4 \mathrm{~ms}^{-1}$

The PVC data in Fig. 3 show a highly reduced scatter, with respect to the Standard results. This finding indicates that the pre-cracks produced by the original sliding technique have poor characteristics. A possible reason for this could be the residual stresses introduced at the crack tip during sliding. Another reason could be that the notches were too shallow, and did not fulfil the recommendation given in the ESIS TC4 protocol for the evaluation of fracture toughness of polymers at slow speed [7]: "The cracks should be several times longer than the pre-notch tip radius". 


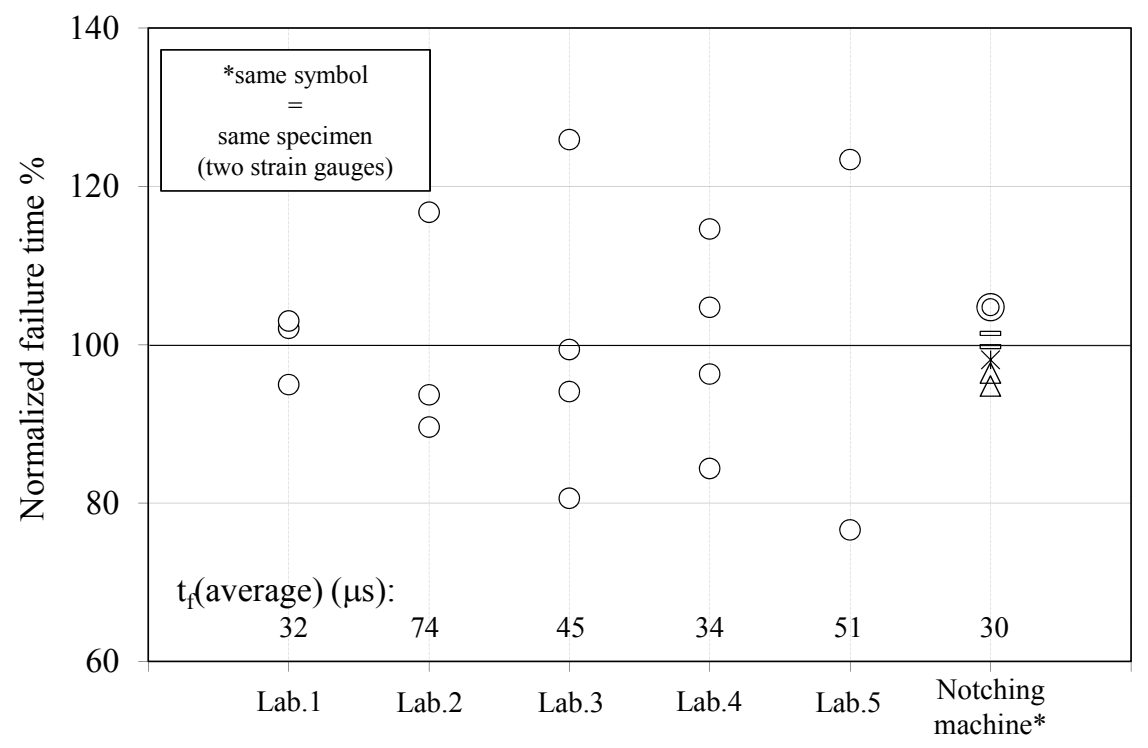

Fig. 8: Normalized time to failure of PMMA samples tested at $4 \mathrm{~ms}^{-1}$

The PMMA Notching machine results in Fig. 4 are characterized by a very high scatter with respect to the Standard results. This finding might be ascribed to the larger notch tip radius of specimens notched by scalpel sliding. However the scalpel sliding technique reduces crack front asymmetry effects: the $t_{f}$ values recorded by strain gauges on the opposite sample faces (see same symbol points) are in good agreement. It must be noticed that this high scatter could be limited by a proper filtering of the data: the points between brackets should not be considered valid data- because a double peak in the strain gauge signal was recorded, corresponding to a crack arrest line on the fractured surface. The same feature was found in some tests of the second round robin, and these results were included in the overall results, thus increasing the data scatter.

On the basis of this investigation, the notching technique turned out to be the factor having the strongest influence on the scatter in time to failure data. Use of a correct notching technique is a vital part of fracture testing. For materials such as PVC which can be cut, then an automated cutting machine which removes material in small steps is the best method of those applied in this work. For harder materials such as PMMA which are not amenable to cutting, a viable method is razor tapping but care must be taken in specimen preparation. It is likely that some specimens will be rejected due to asymmetric or out of plane pre cracks when razor cutting is used.

Since the protocol applied here was developed for high loading rates, some explorative tests were run at $4 \mathrm{~ms}^{-1}$ as well. Sample preparation was the same as used in Notching machine data set at 1 $\mathrm{ms}^{-1}$. Fig. 7 and 8 show the results of PVC and PMMA specimens respectively, at $4 \mathrm{~ms}^{-1}$, compared to those obtained in the round robin activity. Surprisingly, the PMMA data scatter was reduced with respect to the scatter of round robin data, while that for the PVC was comparable. It seems that at 
this speed the material is less notch sensitive, therefore it might be that other factors have a major influence on the data scatter at such high speed. Leevers [8] has proposed a thermal decohesion mechanism for the fracture of some polymers at high strain rates and this mechanism might reduce notch sensitivity as it becomes dominant. However, in consideration of the complexity of the impact phenomena, further work is necessary to fully understand this result.

\section{Conclusions}

In this work the protocol which is being developed by ESIS TC4 to measure fracture toughness for polymers at high speed [6] was applied at $1 \mathrm{~ms}^{-1}$ in order to determine the reason for the high scatter in the failure time data produced in the last round robin activity. High speed camera videos showed that the time to fracture data measured from strain gauge signals was reliable. The same scatter in the round robin results could not be reproduced by intentionally introducing in each test a single experimental source of error, therefore a synergy of these factors is suspected. The parameter with the greatest influence was found to be the notching technique Two modifications could be suggested to improve the protocol, depending on the material tested. i) For relatively tough materials, like PVC, the pre-crack should be several times longer than the notch tip radius and if the sliding technique is used, then it must remove material from the notch. ii) For brittle materials, like PMMA, tapping technique can be used, but data must be filtered a posteriori to exclude results from specimens with asymmetric crack front or those having multiple-peak strain signals.

\section{References}

[1] ISO 17281, Plastics - Determination of fracture toughness (GIC and KIC) at moderately high loading rates $\left(1 \mathrm{~ms}^{-1}\right), 2002$.

[2] Böhme W. Application of dynamic key curves for the determination of the impact fracture toughness of polymers at high rates of loading. In: Williams JG, Pavan A, editors. Impact and dynamic fracture of polymers and composites, ESIS Publication 19, London: Mechanical Engineering Publications; 1995, p. 59-71.

[3] Srawley JE. Wide - range stress intensity factor expressions for ASTM E 399 standard fracture toughness specimens. Int J Fract 1976;12(3):475-80.

[4] Bucci RJ, Paris PC, Landes JD, Rice JR. J integral estimation procedures. In: Fracture Toughness, ASTM STP 514; Philadelphia: ASTM; 1973, p. 40-69.

[5] Williams JG, Tropsa V, MacGillivray H, Rager A. Dynamic correction factors for K and G in high rate, SENB, impact tests. Int J Fract 2001;107(3):259-78. 
[6] Rager A. Analisys of high rate fracture tests of polymers. PhD Thesis, Imperial College, London 2003.

[7] Williams JG. $K_{C}$ and $G_{C}$ at slow speeds for polymers. In: Moore DR, Pavan A, Williams JG, editors. Fracture mechanics testing methods for polymers adhesives and composites, ESIS Publication 28, Oxford: Elsevier; 2001, p. 11-26.

[8] Leevers PS. Impact and dynamic fracture of tough polymers by thermal decohesion in a Dugdale zone. Int J Fract 1995;73(2):109-27. 